\title{
Video Article \\ The Crossmodal Congruency Task as a Means to Obtain an Objective Behavioral Measure in the Rubber Hand Illusion Paradigm
}

\author{
Regine Zopf ${ }^{1}$, Greg Savage ${ }^{2,3}$, Mark A. Williams ${ }^{1,2}$ \\ ${ }^{1}$ Department of Cognitive Science, Macquarie University \\ ${ }^{2}$ ARC Centre of Excellence in Cognition and its Disorders, Macquarie University \\ ${ }^{3}$ Department of Psychology, Macquarie University
}

Correspondence to: Regine Zopf at regine.zopf@mq.edu.au

URL: https://www.jove.com/video/50530

DOI: doi: $10.3791 / 50530$

Keywords: Behavior, Issue 77, Neuroscience, Neurobiology, Medicine, Anatomy, Physiology, Psychology, Behavior and Behavior Mechanisms, Psychological Phenomena and Processes, Behavioral Sciences, rubber hand illusion, crossmodal congruency task, crossmodal congruency effect, multisensory processing, body ownership, peripersonal space, clinical techniques

Date Published: $7 / 26 / 2013$

Citation: Zopf, R., Savage, G., Williams, M.A. The Crossmodal Congruency Task as a Means to Obtain an Objective Behavioral Measure in the Rubber Hand Illusion Paradigm. J. Vis. Exp. (77), e50530, doi:10.3791/50530 (2013).

\section{Abstract}

The rubber hand illusion $(\mathrm{RHI})$ is a popular experimental paradigm. Participants view touch on an artificial rubber hand while the participants' own hidden hand is touched. If the viewed and felt touches are given at the same time then this is sufficient to induce the compelling experience that the rubber hand is one's own hand. The RHI can be used to investigate exactly how the brain constructs distinct body representations for one's own body. Such representations are crucial for successful interactions with the external world. To obtain a subjective measure of the RHI, researchers typically ask participants to rate statements such as "I felt as if the rubber hand were my hand". Here we demonstrate how the crossmodal congruency task can be used to obtain an objective behavioral measure within this paradigm.

The variant of the crossmodal congruency task we employ involves the presentation of tactile targets and visual distractors. Targets and distractors are spatially congruent (i.e. same finger) on some trials and incongruent (i.e. different finger) on others. The difference in performance between incongruent and congruent trials - the crossmodal congruency effect (CCE) - indexes multisensory interactions. Importantly, the CCE is modulated both by viewing a hand as well as the synchrony of viewed and felt touch which are both crucial factors for the RHI.

The use of the crossmodal congruency task within the RHI paradigm has several advantages. It is a simple behavioral measure which can be repeated many times and which can be obtained during the illusion while participants view the artificial hand. Furthermore, this measure is not susceptible to observer and experimenter biases. The combination of the RHI paradigm with the crossmodal congruency task allows in particular for the investigation of multisensory processes which are critical for modulations of body representations as in the RHI.

\section{Video Link}

The video component of this article can be found at https://www.jove.com/video/50530/

\section{Introduction}

We demonstrate how an objective measure of effects in the commonly employed rubber hand illusion paradigm can be obtained by combining this paradigm with the well-established crossmodal congruency task.

In the RHI paradigm participants view touch on an artificial rubber hand while the participants' own hidden hand is touched. If the viewed and felt touches are given at the same time then this is sufficient to induce the compelling experience that the rubber hand is one's own hand for the majority of participants. When touch is given asynchronously then the RHI is abolished or reduced. As the name suggests, the RHI paradigm typically involves a hand, however similar paradigms have also been established for whole bodies ${ }^{1-3}$. Experiments using this paradigm can explore the conditions which modulate body representations. Previous experiments for example demonstrated that both viewing a body form as well as the synchrony of the given touch are important cues for the representation of one's own body ${ }^{4,5}$. Thus, the RHI as an experimental paradigm can be used to shed light on how the brain constructs and updates distinct representations for one's own body ${ }^{4-7}$. Such body representations support many vital processes when interacting with the external environment. Furthermore, body representation changes are related to many clinical disorders for example chronic pain, eating disorders and schizophrenia ${ }^{8-10}$.

To investigate the mechanisms underlying the construction of distinct body representations researchers have employed several measures that are modulated in the rubber hand illusion paradigm. Typically, researchers ask participants to rate statements such as "I felt as if the rubber hand were my hand." on a scale from -3 to $3^{7,11}$. Another more indirect measure which has often been employed involves asking participants to indicate the position of their own hand before and after the illusion was induced ${ }^{12}$. The hand position is typically perceived to be closer to the rubber hand after the $\mathrm{RHI}$ is induced ("proprioceptive drift"). 
Researchers have also employed physiological measures to index the effect of experimental manipulations in the RHI, for example skin conductance responses when a sudden threat (for example an approaching knife) to the rubber hand is perceived ${ }^{13,14}$. The RHI has also been related to small skin temperature changes which can be measured ${ }^{15}$. The advantage of these physiological measures is that they occur automatically and thus are less susceptible to potential experimental biases. Potential disadvantages of these techniques include adaption of the physiological response throughout the experimental manipulation. Furthermore, the physiological processes these measures tap into can be generally deregulated in certain patient groups (for example chronic pain, eating disorders and schizophrenia, see supplementary material in ${ }^{15}$ ).

Our specific aim was to obtain an objective behavioral measure of the effects in the rubber hand illusion, which is less susceptible to possible observer or experimenter biases. For this purpose we combined the RHI with the crossmodal congruency task. This task involves speeded forced-choice location discriminations of targets in one modality while, additional stimuli are presented in a different modality ${ }^{16,17}$. This measure thus involves a relatively simple task which unlike physiological measures can be repeatedly administered many times. Furthermore, unlike "proprioceptive drift" this measure can be obtained on-line during the illusion while participants view the artificial hand. And the combination of the RHI paradigm with the crossmodal congruency task allows in particular for the investigation of multisensory processes which are critical for modulations of body representations as in the $\mathrm{RHI}^{4,5,18}$. We think that the CCE measure is suited to study changes in body representations in patient groups. We think this is especially the case for the study of disorders which include a range of cognitive, motivational or physiological changes which might generally affect more subjective and physiological measures of the RHI.

In the variant we use for the RHI, tactile targets (short vibrations) are presented to different fingers (for example index and middle finger) of one hand. Participants are simply asked to indicate which finger received the tactile stimulation by pressing one of two buttons with the free hand. The distractors are visual stimuli (brief flickering of small lights) which are mounted above the fingers of the viewed rubber hand. These visual stimuli occur close in time to the tactile stimuli. Importantly, the visual stimuli appear on the same finger - spatially congruent - half of the time and occur on the other finger - spatially incongruent - the other half. Spatially congruent visual stimuli improve the localization of the tactile target, whereas spatially incongruent visual stimuli can slow this process down. The overall difference in performance between incongruent and congruent trials, known as the crossmodal congruency effect (CCE), reflects the influence of the visual information on discriminating the tactile target locations and thus indexes multisensory interactions.

Viewing a hand form ${ }^{19-21}$ as well as the synchrony of the given touch ${ }^{22}$ in the RHI determines the magnitude of the CCE. In other words, the CCE magnitude indexes how much the visual stimuli near the rubber hand influence the response to touches on one's own hand. When participants experience the rubber hand to be one's own hand then the CCE for visual-tactile stimuli is increased. It is thought that the RHI leads to changes in multisensory processing which likely increases the interactions between tactile and visual stimuli ${ }^{5,18}$.

We have previously used the combination of the $\mathrm{RHI}$ and crossmodal congruency task to investigate experimental effects in the $\mathrm{RHI}{ }^{22}$. Others have shown that the CCE magnitude can be used as a measure in whole body illusions which involve more global aspects of body representations ${ }^{23}$. In this study participants discriminated the location of tactile vibration stimuli on their backs. At the same time participants viewed their bodies from a position as if standing $2 \mathrm{~m}$ behind their own body via a camera and a head mounted display. Participants could also see lights which were either flashed in the same location as the tactile targets (congruent trials) or in a different location (incongruent trials). In addition to the crossmodal congruency task, the authors also stroked the backs of the participants. This stroking was either in synchrony with viewed strokes or in asynchrony. This manipulation caused on average a difference in the experience of body ownership and also affected the magnitude of the CCE. Thus, the CCE magnitude can be used as an objective measure for changes both in the RHI as well as in the 'whole body illusion'. Combinations of these paradigms with the crossmodal congruency task allow, in particular, probing of multisensory processes which are critical for the occurrence of these illusions. We will now provide a detailed step-by-step description of how we implemented the crossmodal congruency task in the RHI paradigm.

\section{Experimental Setup}

\section{Material and Setup for the Rubber Hand Illusion Setup}

1. Use a box for the RHI paradigm which includes a cover for the participants own hand. Use a gown to also cover the shoulder and arm of the participant as well as the part of the rubber hand which goes up to the wrist.

2. Obtain an artificial hand. This hand does not necessarily have to be made out of rubber (the name 'rubber hand illusion' was given because a rubber hand was used in seminal work ${ }^{7}$ ). For example prostheses can be used.

3. Use two soft brushes to deliver brush strokes in the RHI

4. Use a metronome, a pre-recorded sound file or experimental software to provide timing signals for the experimenter who delivers the brush strokes.

\section{Experimental Setup - Material and Setup for the Crossmodal Congruency Task}

1. For the crossmodal congruency task, use tactors to deliver tactile stimuli. For example small speakers, bone conductors (from hearing aids) or electromagnetic solenoid-type stimulators can be employed. Use 'dummy tactors' for the rubber hand.

2. Control tactile stimulus delivery via the computer sound output, via an interface card or TTL pulses.

3. Fix the position of the speakers in the box.

4. Use for example light emitting diodes (LEDs) to deliver visual stimuli. These can be controlled with TTL pulses from a computer parallel card and powered for example using an USB port or batteries.

5. Use experimental software to program stimulus presentation.

6. Block any potential sound which could arise during the delivery of tactile stimuli. For example use headphones and white noise.

7. Record responses for example using a response box, a computer mouse or a keyboard. 


\section{Participants}

1. All aspects of the experiment are in accord with the ethical standards laid down in the 1964 Declaration of Helsinki and have been approved by our local ethics committee (Macquarie University Human Research Ethics Committee, Australia).

2. Obtain written informed consent for participation prior to the start of the experiment.

3. Participants with visual or tactile deficits especially for the stimulated locations need to be excluded.

\section{Experimental Procedure}

1. Ensure that participants are sitting in a comfortable position.

2. Ensure participants can see the rubber hand and not their own hand.

3. Include training blocks so participants become familiar with the crossmodal congruency task. Ideally first use practice trials without crossmodal visual stimuli in order to accustom participants to the tactile targets and then use practice trials with visual stimulus presentation and no-go trials (see below).

4. On each crossmodal congruency trial present one tactile stimulus and one visual stimulus to one of the locations, either in spatially congruent locations (same fingers) or incongruent locations (different fingers).

5. Instruct participants to respond and discriminate the location of the tactile targets as fast as and as accurately as possible.

6. Locate a fixation point between the lights which participants should fixate throughout the experiment.

7. Use no-go trials in order to ensure that participants are looking at the stimuli. For example ask participants to withhold their response if the lights in both locations turn on at the same time. When participants regularly press a button during these trials, then this can indicate that they are not looking at the lights.

8. Use supra-threshold stimuli and make sure participants can see/feel the stimuli.

9. Introduce a short delay $(150-250 \mathrm{msec})$ between visuotactile crossmodal stimuli in order to reduce interference of these stimuli on the RHI itself.

10. Include sufficient number of congruent and incongruent trials for each condition (about 60-100 trials).

11. Present congruent and incongruent trials in different locations in a randomized sequence.

12. Keep in mind that response requirements and mappings might affect the CCE magnitude ${ }^{24}$

13. In order to induce the RHI use brush stroking for 1 to 3 min before the crossmodal congruency task and then stroke once before every crossmodal congruency trial.

14. To induce the RHI illusion use synchronous brush stroking, where both hands should be touched at the same time and in the same location.

15. Use asynchronous stroking as a control condition, where both hands should be stroked with a temporal offset.

16. Make sure the experimenter can comfortably perform the stroking.

17. You can also use RHI rating scales to monitor subjective experiences during the RHI paradigm. Some researcher suggest to use both objective and subjective measures for the rubber hand illusion paradigm for example ${ }^{25}$. Obtain the rating responses after the task block if you would like to compare it to CCE effects because the task itself may modulate the RHI experience.

\section{Data Analysis}

1. Trials in which participants responded too soon (e.g. faster than $200 \mathrm{msec}$ after stimulus onset) or too slow (e.g. later than $1,500 \mathrm{msec})$ should be discarded from the analysis: check for potential significant differences between conditions for number of discarded trials.

2. Analyze the number of false alarms in no-go trials: check for potential significant differences between conditions for number of false alarms.

3. Obtain the average or median for performance measures (response time for speed and response error for accuracy) for each participant and each condition.

4. Only use response times for correct trials.

5. It is also possible to combine response time and error to a single measure: Inverse Efficiency (IE) $=$ Response time/Error ${ }^{17}$.

6. Calculate and depict crossmodal congruency effects = performance on incongruent minus congruent trials for every trial and condition

7. Use statistical tests in order to assess the statistical significance of condition differences (e.g. ANOVA, t-tests).

\section{Representative Results}

The magnitude of the CCE is significantly modulated in the RHI. In Figures $\mathbf{2}$ and $\mathbf{3}$ we present representative results from a previous study we have conducted ${ }^{22}$. In Figure 2 both the CCE for response time and response error differed significantly between different RHI conditions. The CCE magnitude was significantly increased when the RHI was induced with synchronous stroking as compared to asynchronous stroking which reduces or abolishes the RHI. In Figure 3, we depict data from an experiment where brush stroking was not delivered prior to every crossmodal congruency task. Instead, brushing was blocked and given prior to an entire larger set of trials. In this design the CCE is not significantly modulated. 


\section{Experimental Setup}

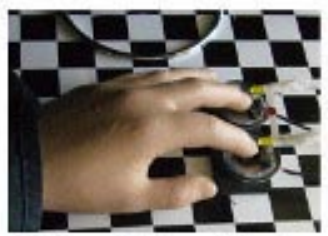

Picture Rubber Hand,

Lights and Tactors

\section{Experimental Procedure}
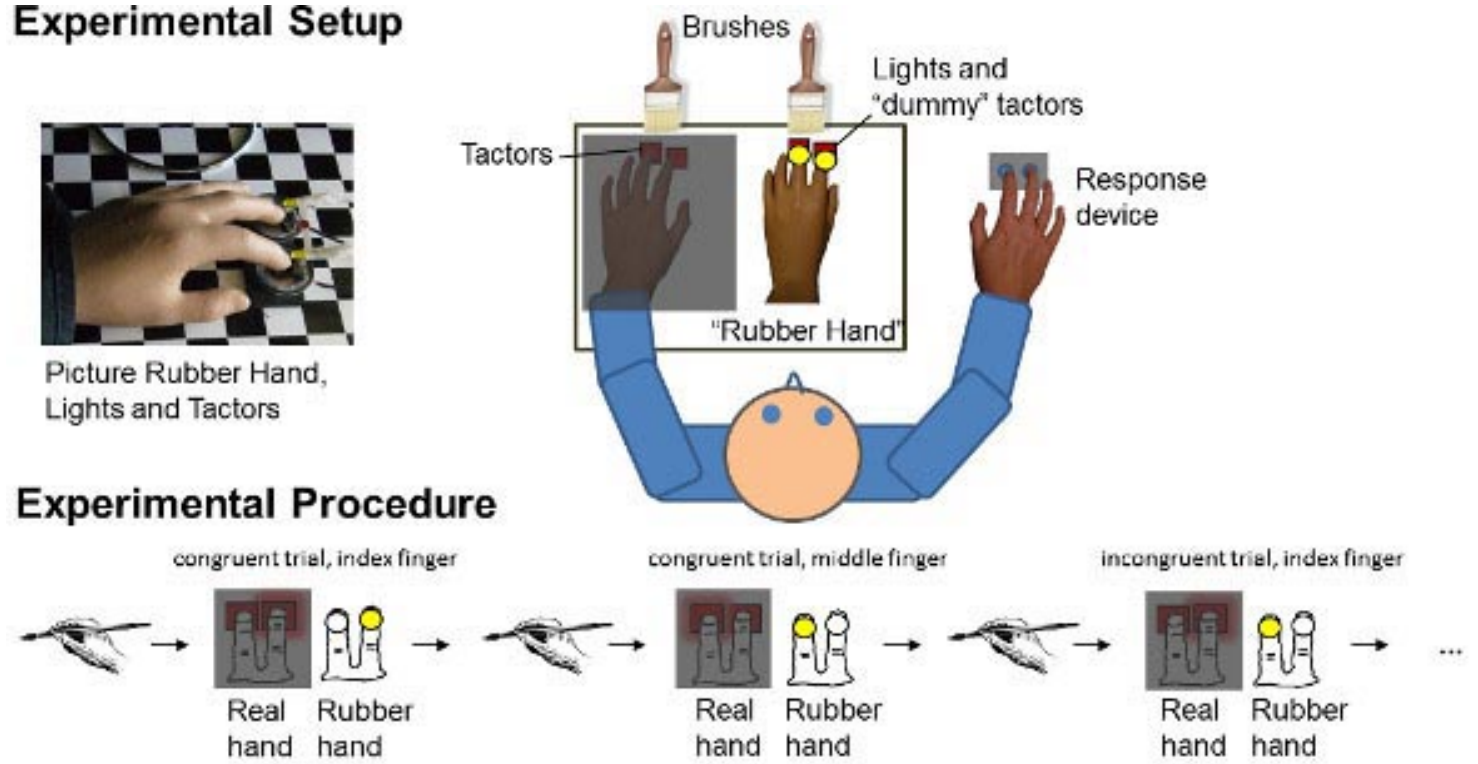

\section{Analysis}

Average or median performance measure for each participant and each condition:

- Response Time (RT in msec), Response Error ( $E$ in \%) or

a combination of these performance measures: Inverse Efficiency $(I E)=R T / E$

- Crossmodal Congruency Effect (CCE) =

Performance Incongruent Trials - Performance Congruent Trials

- Statistical Analyses (e.g. ANOVA or t-tests)

Figure 1. Overview setup and procedure. The experimental setup includes a cover for the participant's real hand, a rubber hand, tactors, lights, brushes and a response device. The covered hand of the participant and the rubber hand are brushed synchronously to induce the RHI. Brush strokes are given prior to every crossmodal congruency trial. The difference in performance between incongruent and congruent trials - the crossmodal congruency effect (CCE) - indexes changes in the RHI. Click here to view larger figure.

A Response time

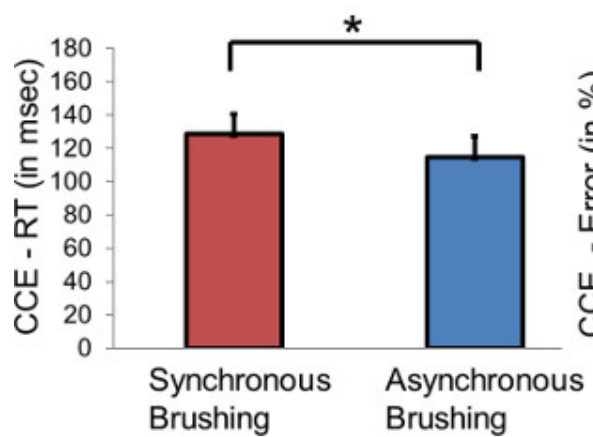

B Response error

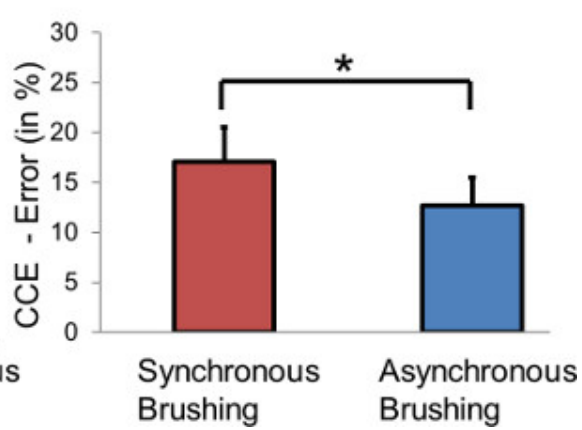

Figure 2. The crossmodal congruency effect (CCE) is shown for both response time and response error. Synchronous brushing (red) induces the $\mathrm{RHI}$ and asynchronous brushing (blue) abolishes the RHI. The crossmodal congruency effect (CCE) for response time and response error differs significantly between synchronous and asynchronous brushing. Adapted from Zopf et al. (2010), with permission (average for synchronous and asynchronous stroking from Experiment 2). 


\section{CCEs when brush stroking was not performed prior to crossmodal congruency trials}

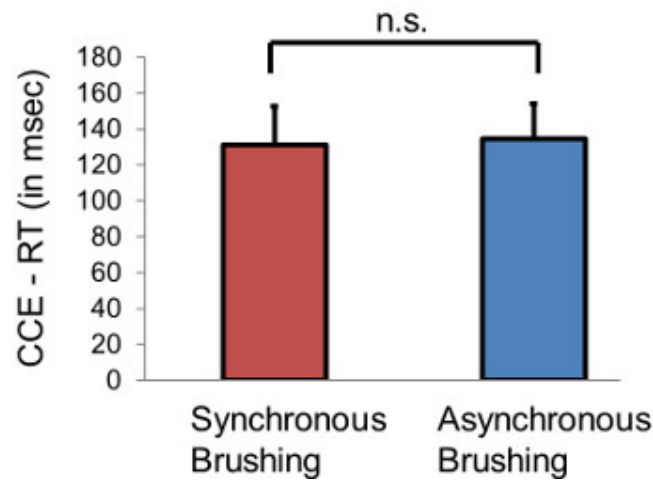

Figure 3. Crossmodal congruency effect (CCE) does not differ significantly between RHI conditions when brush stroking is not performed prior to crossmodal congruency trials and in this case only as one block before a set of trials. Adapted from Zopf et al. (2010), with permission (average for synchronous and asynchronous stroking from Experiment 1).

\section{Discussion}

We demonstrated how an objective measure of effects in the commonly employed RHI paradigm can be obtained by combining this paradigm with the well-established crossmodal congruency task. The magnitude of the crossmodal congruency effect is significantly increased when the $\mathrm{RHI}$ is induced.

Stimulation as given in the rubber hand illusion paradigm modulates several aspects of body-related experiences such as body-ownership and body-location. Research has provided evidence that several processes related to perception, homeostasis and action are modulated in the $\mathrm{RH}$ $14,15,22,26,27$. Importantly, the combination of the RHI paradigm with the crossmodal congruency task allows in particular for the investigation of multisensory processes which are critical for all of these aspects.

Both viewing of the hand and the synchrony of stroking in the RHI, as well as in the whole body illusion, has been shown to also modulate the magnitude of the CCE ${ }^{20,22,23}$. The crossmodal congruency task provides a relatively simple on-line measure which can be repeated many times and is not susceptible to observer and experimental biases.

Potential disadvantages of using this task are that it can be more time consuming and can be technically more sophisticated as compared to other measures. Furthermore, measuring the effects of the RHI can itself modulate the illusion. The important challenge for the crossmodal congruency task is that it involves multisensory visual and tactile stimuli, which can independently of brush stroking modulate body representation changes. The crossmodal congruency task itself might thus interfere with the effect of brush stroking in the RHI. We found that stroking prior to each crossmodal congruency trial is more effective than just stroking at the beginning of a whole set of trials ${ }^{22}$. Furthermore, Aspell et al. (2009) found that presenting the visual and tactile stimuli for the crossmodal congruency task further apart (for example with a small delay of $150-250 \mathrm{msec}$ ) increases the effects of prior stroking. Such a small delay still allows for multisensory interactions ${ }^{28}$, but reduces the interference of visuo-tactile stimuli on the RHI manipulation. Furthermore, we found that the effect of stroking synchrony is reduced when using the crossmodal congruency task while at the same time placing the rubber hand relatively close to the real hand ${ }^{22}$. Most likely this is partly due to a relatively long stimulation interval (up to $15 \mathrm{~min}$ ) and possibly partly because the asynchronous condition also includes crossmodal visuotactile stimulation making this condition "more synchronous" or "less asynchronous" than it usually would be. Therefore, we would recommend using a larger distance between the hands when implementing this method. All in all, the multisensory stimuli in the crossmodal congruency task can itself modulate body representations by changing the amount of overall synchronous or asynchronous multisensory stimulation. When comparing synchronous with asynchronous stimulations in the illusion paradigms, it is thus important to ensure that the brush stroking leads to relatively more overall synchronous or asynchronous visual-tactile stimulation. This can be achieved by trial-by-trial brush stroking and using a short delay between visuotactile crossmodal congruency task stimuli.

Our previous study demonstrated the effect of RHI manipulations on CCE ${ }^{22}$. However, other aspects which have been studied for other RHI measures still need to be investigated. For example, it would be interesting to study if the degree of the RHI for example of the experience of ownership is related to CCE magnitude and if the CCE magnitude changes over time. Furthermore, it would be interesting to investigate if the CCE effects during the RHI are comparable to results observed with the same task on the real hand.

To summarize, we demonstrated that the crossmodal congruency task can be used to obtain an objective behavioral measure in the rubber hand illusion. This behavioral task is relatively simple and we think it is suitable for studying changes in body representations and underlying multisensory processing in both the general population as well as patient groups. However, it is important to note that this measure might not be causally related to subjective experiences in the rubber hand illusion ${ }^{29}$. It is likely that both objective and subjective measures rely on common or similar multisensory mechanisms but possibly also on separate mechanisms. Depending on the research question, it might be advisable to obtain an objective behavioral measure and also a more subjective measure using rating scales. 


\section{Disclosures}

The authors declare that they have no competing financial interests.

\section{Acknowledgements}

M.A.W. is funded by a Queen Elizabeth II Fellowship (DP 0984919) from the Australian Research Council.

\section{References}

1. Ehrsson, H.H. The experimental induction of out-of-body experiences. Science (New York, N.Y.). 317, 1048 (2007).

2. Lenggenhager, B., Tadi, T., Metzinger, T., \& Blanke, O. Video ergo sum: manipulating bodily self-consciousness. Science (New York, N.Y.). 317, 1096-1099 (2007)

3. Petkova, V.I. \& Ehrsson, H.H. If I were you: perceptual illusion of body swapping. PloS one. 3, e3832 (2008).

4. Tsakiris, M. My body in the brain: a neurocognitive model of body-ownership. Neuropsychologia. 48, 703-712 (2010).

5. Ehrsson, H.H. In: The New Handbook of Multisensory Processing., Stein, B.E., ed., MIT Press, (2012).

6. Blanke, O. Multisensory brain mechanisms of bodily self-consciousness. Nature Reviews Neuroscience., doi:10.1038/nrn3292 (2012).

7. Botvinick, M. \& Cohen, J. Rubber hands 'feel' touch that eyes see. Nature. 391, 756 (1998).

8. Eshkevari, E., Rieger, E., Longo, M.R., Haggard, P., \& Treasure, J. Increased plasticity of the bodily self in eating disorders. Psychol. Med., 1-10 (2011)

9. Moseley, G.L., Gallace, A., \& Spence, C. Bodily illusions in health and disease: physiological and clinical perspectives and the concept of a cortical 'body matrix'. Neuroscience and Biobehavioral reviews. 36, 34-46 (2012).

10. Thakkar, K.N., Nichols, H.S., McIntosh, L.G., \& Park, S. Disturbances in body ownership in schizophrenia: evidence from the rubber hand illusion and case study of a spontaneous out-of-body experience. PloS one. 6, e27089 (2011).

11. Longo, M.R., Schuur, F., Kammers, M.P., Tsakiris, M., \& Haggard, P. What is embodiment? A psychometric approach. Cognition. 107, 978-998 (2008).

12. Tsakiris, M. \& Haggard, P. The rubber hand illusion revisited: Visuotactile integration and self-attribution. Journal of Experimental Psychology: Human Perception and Performance. 31, 80-91 (2005).

13. Armel, K.C. \& Ramachandran, V.S. Projecting sensations to external objects: evidence from skin conductance response. Proceedings of the Royal Society of London Series B: Biological Sciences. 270, 1499-1506 (2003).

14. Ehrsson, H.H., Wiech, K., Weiskopf, N., Dolan, R.J., \& Passingham, R.E. Threatening a rubber hand that you feel is yours elicits a cortical anxiety response. Proceedings of the National Academy of Sciences of the United States of America. 104, 9828-9833 (2007).

15. Moseley, G.L., et al. Psychologically induced cooling of a specific body part caused by the illusory ownership of an artificial counterpart. Proceedings of the National Academy of Sciences of the United States of America. 105, 13169-13173 (2008).

16. Spence, C., Pavani, F., Maravita, A., \& Holmes, N. Multisensory contributions to the 3-D representation of visuotactile peripersonal space in humans: evidence from the crossmodal congruency task. Journal of Physiology (Paris). 98, 171-189 (2004).

17. Spence, C., Pavani, F., Maravita, A., \& Holmes, N.P. In: Haptic rendering: Foundations, algorithms, and applications., Lin, M.C., Otaduy, M.A., eds., AK Peters, 21-52 (2008).

18. Makin, T.R., Holmes, N.P., \& Ehrsson, H.H. On the other hand: Dummy hands and peripersonal space. Behavioural Brain Research. 191, 1-10 (2008)

19. Austen, E.L., Soto-Faraco, S., Enns, J.T., \& Kingstone, A. Mislocalizations of touch to a fake hand. Cognitive, Affective \& Behavioral Neuroscience. 4, 170-181 (2004).

20. Pavani, F., Spence, C., \& Driver, J. Visual capture of touch: out-of-the-body experiences with rubber gloves. Psychological Science. 11, 353-359 (2000).

21. Walton, M. \& Spence, C. Cross-modal congruency and visual capture in a visual elevation-discrimination task. Experimental Brain Research. 154, 113-120 (2004).

22. Zopf, R., Savage, G. \& Williams, M.A. Crossmodal congruency measures of lateral distance effects on the rubber hand illusion. Neuropsychologia. 48, 713-725 (2010).

23. Aspell, J.E., Lenggenhager, B., \& Blanke, O. Keeping in touch with one's self: multisensory mechanisms of self-consciousness. PloS one. 4, e6488 (2009).

24. Gallace, A., Soto-Faraco, S., Dalton, P., Kreukniet, B., \& Spence, C. Response requirements modulate tactile spatial congruency effects. Experimental Brain Research. Experimentelle Hirnforschung. 191, 171-186 (2008).

25. Kalckert, A. \& Ehrsson, H.H. Moving a Rubber Hand that Feels Like Your Own: A Dissociation of Ownership and Agency. Frontiers in Human Neuroscience. 6, 40 (2012).

26. Zopf, R., Harris, J.A., \& Williams, M.A. The influence of body-ownership cues on tactile sensitivity. Cognitive Neuroscience. 2, 147-154 (2011).

27. Zopf, R., Truong, S., Finkbeiner, M., Friedman, J., \& Williams, M.A. Viewing and feeling touch modulates hand position for reaching. Neuropsychologia. 49, 1287-1293 (2011).

28. Shore, D.I., Barnes, M.E., \& Spence, C. Temporal aspects of the visuotactile congruency effect. Neuroscience Letters. 392, 96-100 (2006).

29. Rohde, M., Di Luca, M., \& Ernst, M.O. The Rubber Hand Illusion: feeling of ownership and proprioceptive drift do not go hand in hand. PloS one. 6, e21659 (2011). 